\title{
Human health risk assessment simulations in a distributed environment for Shuttle launch
}

\author{
Rajkumar Thirumalainambi ${ }^{a}$ and Jorge Bardina ${ }^{b}$ \\ a SAIC @ NASA Ames Research Center, Mail Stop 269-2, Moffett Field, California, USA; \\ ${ }^{b}$ NASA Ames Research Center, Mail Stop 269-2, Moffett Field, California, USA
}

\begin{abstract}
During the launch of a rocket under prevailing weather conditions, commanders at Cape Canaveral Air Force station evaluate the possibility of whether wind blown toxic emissions might reach civilian and military personnel in the near by area. In our model, we focused mainly on Hydrogen chloride $(H C L)$, Nitrogen oxides $\left(N O_{x}\right)$ and Nitric acid $\left(\mathrm{HNO}_{3}\right)$, which are non-carcinogenic chemicals as per United States Environmental Protection Agency (USEPA) classification. We have used the hazard quotient model to estimate the number of people at risk. It is based on the number of people with exposure above a reference exposure level that is unlikely to cause adverse health effects. The risk to the exposed population is calculated by multiplying the individual risk and the number in exposed population. The risk values are compared against the acceptable risk values and GO or NO-go situation is decided based on risk values for the Shuttle launch. The entire model is simulated over the web and different scenarios can be generated which allows management to choose an optimum decision.
\end{abstract}

Keywords: Human health risk, Shuttle Launch, Virtual test bed, Modeling and Simulation

\section{INTRODUCTION}

Humans are exposed daily to various concentrations of many natural and artificial chemical substances. Although many of these substances can be toxic at high concentrations, typical exposures are far below the concentrations where effects are obvious. However, the long term cumulative effects can be very real, and the identification of these adverse effects has stimulated the development of a scientific approach to risk assessment. Exposure/dose assessment is a central component of the quantitative risk assessment procedure. Traditional risk assessment procedures are based on Monte Carlo simulation. The steps involved in Risk assessment ${ }^{1}$ are (i) Hazard identification, (ii) Dose response assessment, (iii) Exposure assessment, (iv) Risk characterization, and (v) Risk management. Risk characterization synthesizes the first three components of the risk assessment process.

Presently, the US Air force has developed a probabilistic health risk model, the Launch Area Toxic Risk Assessment (LATRA) ${ }^{2}$ model to assist commanders in determining the risks to military personnel and civilians from exposure to emissions from normal and failed launches. The model estimates the mean number of persons who might experience mild or serious health problems. LATRA deals with toxicity of three major rocket emissions: $\mathrm{HCL}, \mathrm{NO}_{2}$ and $\mathrm{HNO}_{3}$. The choice of exposure-response model for each emission is lognormal or probit functions.

\section{DEVELOPMENT OF LATRA}

The rocket exhaust effluent diffusion model $\left(\mathrm{REEDM}^{3}\right)$ simulates the dispersion of a rocket's emission under prevailing weather conditions. REEDM predicts an isopleth of the concentrations of specific gas at ground level downwind of launch pad. Initially, the Air Force compared the exposure concentrations predicted by REEDM for each of the emissions with acceptable human exposure levels, called tier limits. ${ }^{2}$ The Air Force adopted a threetiered concept to delineate acceptable exposure concentrations and durations for the public (tier 1), government and contractor personnel on the ranges (tier 2) and operational personnel directly involved with the launch (tier 3). The Air Force defines the tier 1 exposure limit (the outermost tier) as the airborne exposure concentration that poses no hazards to the general population but might affect sensitive population (e.g., individuals with

Send correspondence to Dr. Jorge Bardina. E-mail: Jorge.E.Bardina@nasa.gov 
asthma or emphysema). The Air Force defines tier 2 exposure limit as the airborne exposure concentration that might cause short-term symptoms that most individuals could endure without experiencing or developing irreversible or other serious health effects or symptoms that could impair their ability to take protective action. The Air Force defines the tier 3 exposure limit as an airborne exposure concentration that can be immediately dangerous to life and health (IDLH). Areas within tier 3 warrant immediate isolation and evacuation actions to prevent exposure. Table (1) indicates three chemical species and their exposure limits. If REEDM predicted

Table 1. Air Force Tier Exposure Limits. ${ }^{2}$

\begin{tabular}{|l|c|c|c|}
\hline & Tier 3 (inner) ppm & Tier 2 (middle) ppm & Tier 1 (outer) ppm \\
\hline $\mathrm{HCL}$ & 50 & 10 & 10 \\
\hline $\mathrm{NO}_{2}$ & 20 & 4 & 2 \\
\hline $\mathrm{HNO}_{3}$ & 25 & 4 & 0.3 \\
\hline
\end{tabular}

that specific populations would be exposed at higher than the appropriate tier limits, the commander would be advised to hold the launch.

The exposure-response functions (ERFs) in LATRA, ${ }^{2},{ }^{4}$ translate exposure estimates from REEDM into probabilities of health effects for specified severity categories in the human population. At present, separate ERFs are developed for two segments of the population: "sensitive" and "normal" populations. The ERFs included in LATRA at present are lognormal for noncarcinogenic substances and linear passing through the origin for carcinogenic substances. For each severity category, the ERF is the probability, $P_{E}$, per individual of an effect, $\gamma$, exceeding in a given severity category, $S$ (mild or serious) given an exposure concentration and duration $\mathrm{T}$ as shown in equation (1).

$$
P_{E}(S, C, T)=P(\gamma \geq S / C, T)
$$

where $P_{E}(S, C, T)$ is the ERF for an exposure characterized by concentration, $\mathrm{C}$, and time or duration of exposure, $T$, and is equal to the probability of the severity equaling or exceeding a given severity category, $S$, at a specified exposure concentration, $\mathrm{C}$, and duration, $\mathrm{T}$.

Sensitive populations are defined as children (less than 15 years of age), the elderly (more than 64 years of age) and all persons with bronchitis, asthma, or other physiological stress, especially upper respiratory ailments. ${ }^{2}$ Under LATRA, the remainder of the population is considered as normal and is assumed to be healthy adults. The LATRA model has three health effect severity levels, as listed below.

- Mild : no damage to body organs; temporary irritation

- Significant (or severe or serious): damage to body organs; treatment required

- Fatal: (considered unacceptable ${ }^{6}$ )

The mild response is identified as a irritation of eyes, skin and upper airways. The significant level is identified as reversible or irreversible response that might require medical intervention, especially when the central airways of the tracheobronchial tree are involved.

The lognormal ERFs for noncarcinogenic rocket emissions are specified at present by two symmetric percentiles.: the 1 and 99 percentiles. The assumption is that the $1 \%$ effect level represents exposure below which "essentially no one" would experience each specifed severity effect. The $99 \%$ effect level represents exposures above which "essentially everyone" would experience the specified severity effect. The $99 \%$ effect levels are set 5-fold higher than the $1 \%$ effect levels for sensitive population and 10 -fold higher than $1 \%$ for normal population. Table (2) describes the exposure concentrations associated with the $1 \%$ and $99 \%$ incidence values for sensitive and normal populations and mild and serious effects for the three rocket emission toxicants. The purpose of the LATRA ERF is to provide a model that links the expected number and severity of effects with both exposure 
Table 2. Exposure concentrations associated with the $1 \%$ and $99 \%$ effect levels in the LATRA-ERFs. ${ }^{2}$

\begin{tabular}{|ll|ll|ll|ll|}
\hline & & \multicolumn{2}{|l|}{ Hcl, ppm } & \multicolumn{2}{|l|}{$\mathrm{NO}_{2}, \mathrm{ppm}$} & \multicolumn{2}{|l|}{$\mathrm{NO}_{3}, \mathrm{ppm}$} \\
\hline Population & Effect Level & $1 \%$ & $99 \%$ & $1 \%$ & $99 \%$ & $1 \%$ & $99 \%$ \\
\hline Sensitive & Mild & 10 & 50 & 2 & 10 & 0.3 & 1.5 \\
\hline & Serious & 20 & 100 & 4 & 20 & 4 & 20 \\
\hline Normal & Mild & 10 & 100 & 4 & 40 & 4 & 40 \\
\hline & Serious & 50 & 500 & 8 & 80 & 8 & 80 \\
\hline
\end{tabular}

concentration and exposure duration. Model selection depends on the quantity and quality of available data. A complex probit model can be provided by equation 2 .

$$
P(\gamma \geq S \mid C, T)=O\left[A+B \times\left(C \times T^{g}\right)\right]
$$

If the exponent ' $\mathrm{g}$ ' equals 1 , it would result in a Haber's rule model. Haber's rule states that the biological effects of some types of toxicants tend to be related to the total cumulative exposure (area under the concentration time curve). For a strict Haber's rule model, the total exposure for risk estimation is the time weighted average concentration (C) multiplied by the time ( $\mathrm{T})$. The ' $\mathrm{O}$ ' designates the cumulative normal distribution. Another model at this level of complexity would be a logistic model, which would take the form in equation 3 .

$$
P(\gamma \geq S \mid C, T)=\exp \left[A+B \times\left(C \times T^{g}\right)\right] /\left[1+\exp \left[A+B \times\left(C \times T^{g}\right)\right]\right]
$$

The probit and logistic models provide similar results in the $1 \%$ to $99 \%$ incidence range. The logistic model is more flexible in handling exposure concentration versus time.

In 1995, the Air Force Air Space command asked the National Research Council (NRC) for an independent review of the ERFs in LATRA. The committee found the basic premise of the LATRA model using exposure versus incidence of response models to predict the incidence of effects in humans to be reasonable, but the available toxicological data on the specified rocket emission toxicants are currently insufficient to support the ERFs used in the LATRA model. The committee found the available toxicity data on $\mathrm{HCl}, \mathrm{NO}_{2}$, and $\mathrm{HNO}_{3}$ are insufficient to support the development of ERFs for mild and serious effects in sensitive and normal human populations. In principle, the LATRA-ERF model is a valid concept, but the subcommittee does not endorse use of the LATRA-ERF model as it is currently constructed. The committee recommended the hazand quotient approach to be used to characterize risks for sensitive and normal populations.

\section{HAZARD QUOTIENT - HAZARD INDEX APPROACH}

The potential noncarcinogenic health effects resulting from exposure to a chemical is generally assessed by comparing an exposure estimate to an $R f D$ for oral exposures and comparing an estimated chemical specific-air concentration to the $R f C$ for direct inhalation exposures. ${ }^{1}$ An $R f D$ is a daily oral intake rate that is estimated to pose no appreciable risk of adverse health effects, even to sensitive populations, over a specific exposure duration. Similarly, an $R f C$ is an estimated daily concentration of a chemical in air, the exposure to which over a specific exposure duration poses no appreciable risk of adverse health effects, even to sensitive populations. The comparison of exposure estimates and specific air concentrations to $R f D$ and $R f C$ values are known as hazard quotient (HQ), which is calculated as follows in equation 4.

$$
H Q=A D D \div R f D \text { or } H Q=C_{a} \div R f C
$$

where $\mathrm{HQ}$ is hazard quotient (unitless), ADD is the average daily dose $(m g / k g-d a y), R f D$ is the reference dose $(m g / k g-d a y), R f C$ is the reference concentration $\left(m g / m^{3}\right)$ and $C_{a}$ is the total chemical concentration 
$\left(m g / m^{3}\right)$. The USEPA calculates ADD by the following equation 5 .

$$
A D D=\frac{C \times I R \times E T \times E D \times E F \times 0.001 \frac{\mathrm{mg}}{\mu g}}{B W \times A T \times 365 \frac{d a y}{\mathrm{yr}}}
$$

where $\mathrm{C}$ is the chemical concentration $\left(\mu \mathrm{g} / \mathrm{m}^{3}\right)$, IR is the inhalation rate $\left(\mathrm{m}^{3} / \mathrm{hr}\right)$, ET is the exposure time (hrs/day), EF is the exposure frequency (days/yr), ED is exposure duration (yr), BW is the body weight (kg) and AT is the averaging time in yr. The US EPA recommends using default values for IR as 0.63 (for adults) and 0.3 (for children) in the absence of site specific information. It also recommends default values for $B W$ as 70 $\mathrm{Kg}$ (for adult) and $15 \mathrm{Kg}$ (for children). The averaging time is expressed as $70 \mathrm{yr}$ (as a life span for an adult).

However, because $R f D s$ and $R f C s$ do not have equal accuracy or precision and are not based on the same severity of effect, the level of concern does not increase linearly as $H Q$ approaches and exceeds 1 . The total noncarcinogenic hazard for each exposure pathway is calculated by following the procedures outlined by the US EPA. Specifically, the total noncarcinogenic hazard attributable to exposure to all chemicals through a single exposure pathway is known as hazard index. The $\mathrm{HI}$ is calculated as follows in equation 6 .

$$
H I=\sum_{i} H Q_{i}
$$

where $\mathrm{HI}$ is the total hazard for a specific exposure pathway and $H Q_{i}$ is the hazard quotient for chemicals $\mathrm{i}$. For the purposes of the risk assessment, it is reasonable to estimate a recipient's total hazard as the sum of the $H I s$ for each of the exposure pathways identified. Specifically, a recipient's total hazard is the sum of hazards from each individual exposure pathway, expressed as follows in equation 7.

$$
\text { Total HI }=\sum H I
$$

where Total $H I$ is the total hazard from multiple exposure pathways and $H I$ is the total hazard for a specific exposure pathway. The hazard quotient approach allows estimation of the number of people at risk from additive effects from simultaneous exposure to two or more substances, a consideration that is not appropriately represented in the LATRA model and that would be difficult to develop in the ERF framework.

\section{OPEN MAP ARCHITECTURE AND HUMAN HEALTH RISK ASSESSMENT MODEL}

The architecture is supported by tomcat web server and openMap ${ }^{7}$ GIS software to connect toxic dispersion model and human health risk assessment model. The openMap interacts at the client side and acts as a graphical user interface. The openMap java toolkit is made up of Java Bean components, of which the MapHandler, MapBean and Layer/PlugIn components play vital roles. The openMap makes it easy to build components that fit the architecture to present user defined data. The MapHandler can be defined as a conceptual map, which contains MapBean and other components that manage the layers, mouse events and projection controls. The MapHandler is really an extended version of Java's BeanContext. The BeanContext is a mechanism that Java Beans can be used to find other beans which can interact with. All openMap components have been designed to use the MapHandler to locate and connect to other components they need. The MapBean is a drawing Canvas that derives from the Swing JComponent class. Because the MapBean is a Swing component, it can be added to a Java window hierarchy like any other swing windowing component. The MapBean manages a hierarchy of layers which can paint themselves to the canvas and a projection object to manage the view. Layers are the only component that can be added to a MapBean. When a Layer is added, it becomes a ProjectionListener to the MapBean, and receives a ProjectionEvent whenever the map is panned, zoomed or resized. In figure (1), there are two layers added namely HRA and TGD layers. The TGD layer invokes gas dispersion model, ${ }^{89}$ from the server by sending a Http request. The HRA layer computes the risk based on available ground gas concentration and population density in a given area. The risk is computed based on equation 4, 5, 6, and 7 .

The openMap Applet depends on the viewer application for rendering images on web browsers. The openMap viewer application uses the BufferedMapBean instead of the MapBean precisely because of the increased 


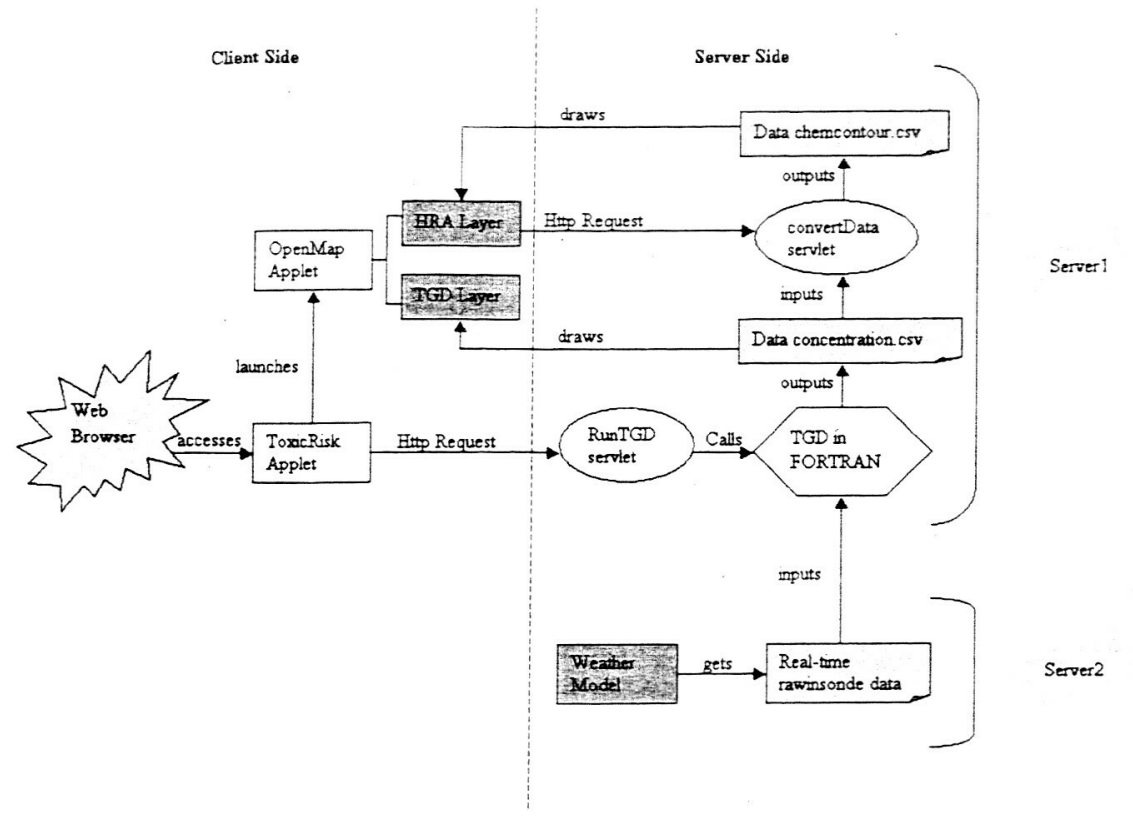

Figure 1. Schematic diagram of http protocol in toxic gas dispersion model.

performance. The BufferedMapBean extends the MapBean by forcing its layers to paint their graphics into a buffer. This drawing buffer is then rendered whenever the AWT causes a redraw. This dramatically increases performance for window exposes since it avoids the layer painting process. Of course if a layer causes a redraw, then it must regenerate the drawing buffer with the graphics and render new image. From the web browser, ToxicRisk applet sends a request to web server, calls dispersion models. For dispersion models, rawinsonde data $^{10}$ are provided from server 2 as inputs. The gas dispersion model performs computations and ground level concentration is provided as an output. This data has been converted to necessary formats, and it forms as an input to the risk assessment model.

\subsection{User Inputs}

The default launch time and date is considered as the system date. The user has an option to edit the launch time and date. The user has an option to select the type of vehicle from among 7 types. Once the user has selected a particular vehicle, the type of fuel, and fuel expenditure determined. The user can select different launch pads available at Cape Canaveral. The user can select chemical species, and their absorption coefficients. The user can use a default rawinsonde or real time rawinsonde data by clicking appropriate check box. The user can specify cloud characteristics. Once the user has provided all the input, the client sends the data to the server and the toxic gas dispersion computes ground level chemical concentration. The user input screen is shown in figure (2). Once the concentration is computed, the risk assessment can be computed by providing the reference concentration, body weight of adult and exposure duration as shown in figure (3). To invoke the risk assessment parameter screen, the user has to go through submenus to access risk assessment layer. In figure (4), the user has to turn on the risk assessment layer. The user can move forward or backward through the layers hierarchy. By default, the normal adults characteristics and $\mathrm{HCl}$ chemical characteristics are assumed. The user can alter the values and can run the model. Once the user closes the risk assessment parameter window, the risk assessment layer computes automatically. 


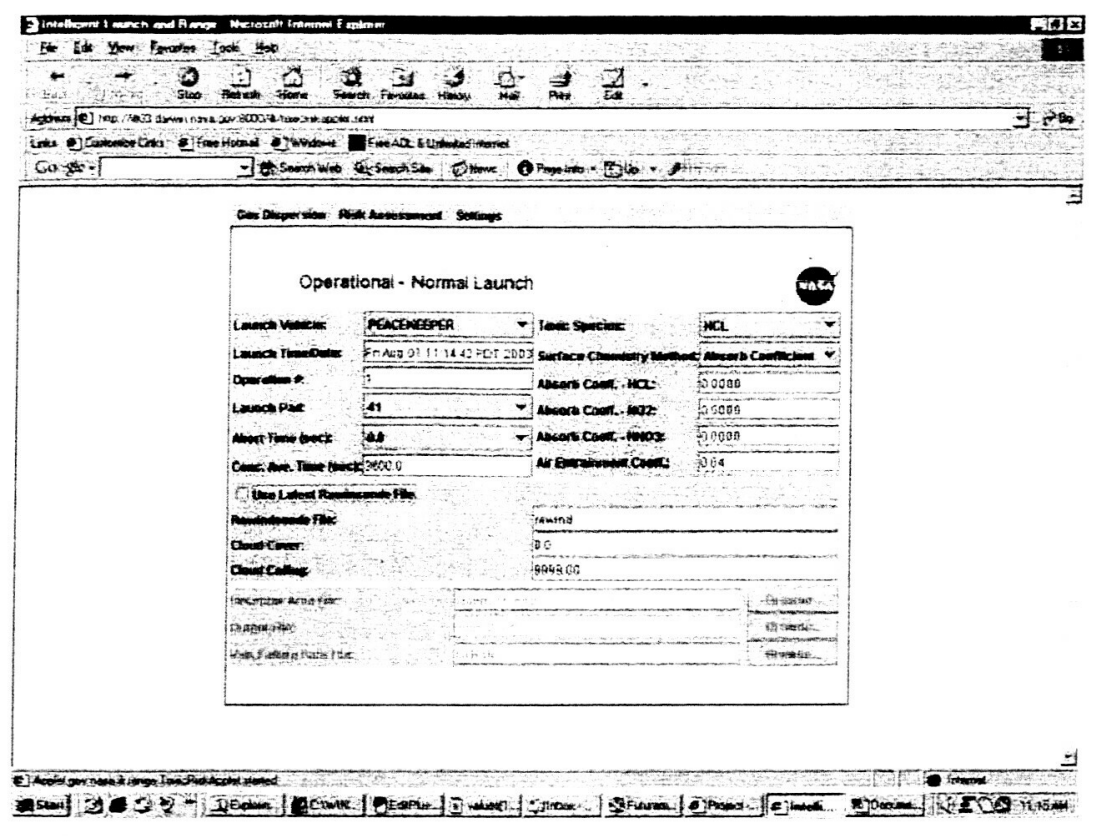

Figure 2. Screen shot of input window for toxic gas dispersion.

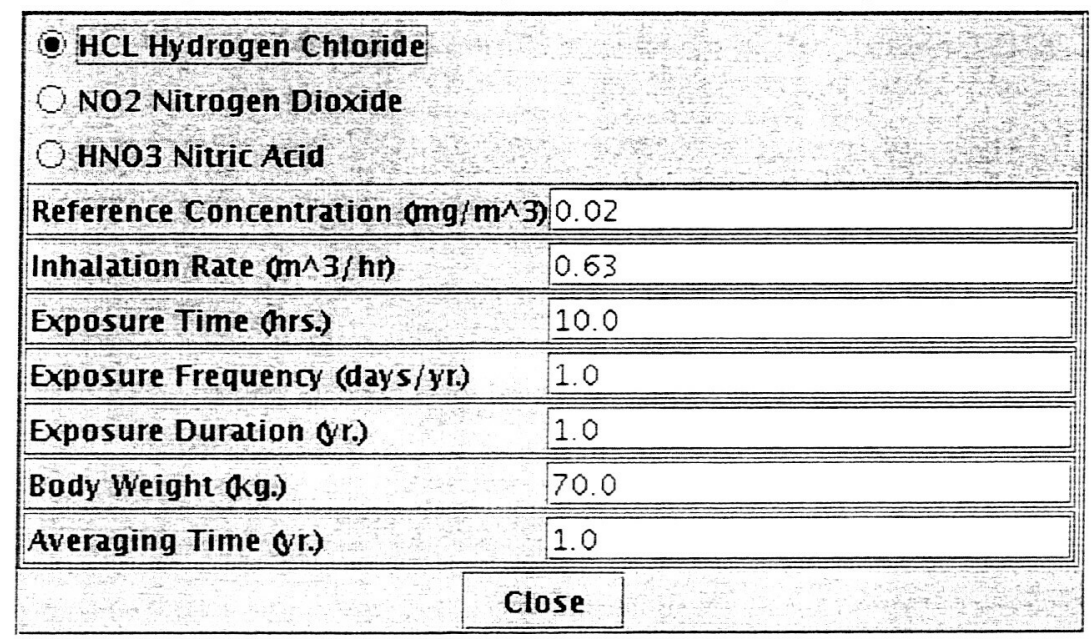

Figure 3. Screen shot of input window for Risk assessment parameters.

\subsection{Outputs}

Figure (4) also shows other layers such as Date and time, Launch Population, Gas concentration, Risk assessment, Gas concentration contour, Population contour, Florida state map, Launch areas, Graticule and chemical concentration contour. Figure (5) shows the chemical concentration contour plot. The openMap viewer is an application that can be easily configured to add and remove components, without code modification. The new components can be added by adding a reference to openmap.properties. The Florida state GIS information is provided via properties file. The user can add/remove layers in the application, turn them on and off. The prop- 


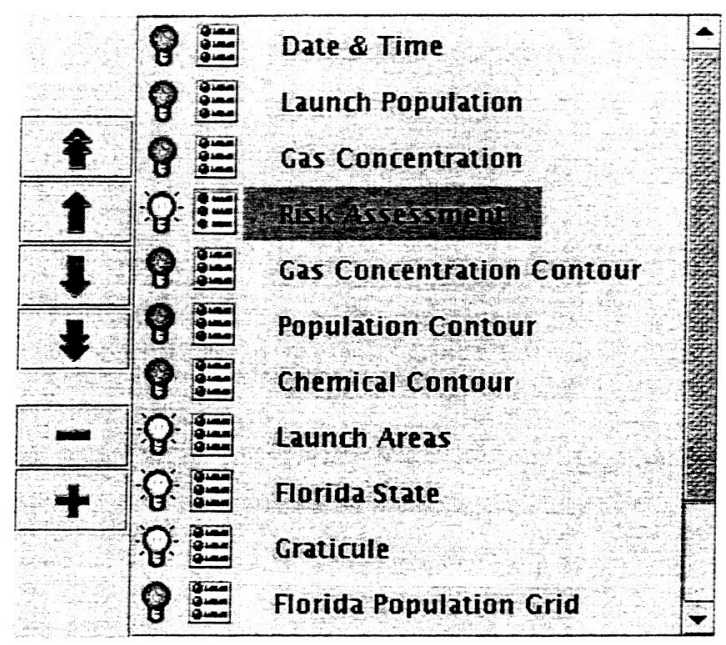

Figure 4. Selection of risk assessment layer from openMap layer Palette.

ertyHandler is a SoloMap component that can search for and parse openmap.properties files, directing certain properties to components that need them. At the top level is the MenuBar, which is an extension of the Java

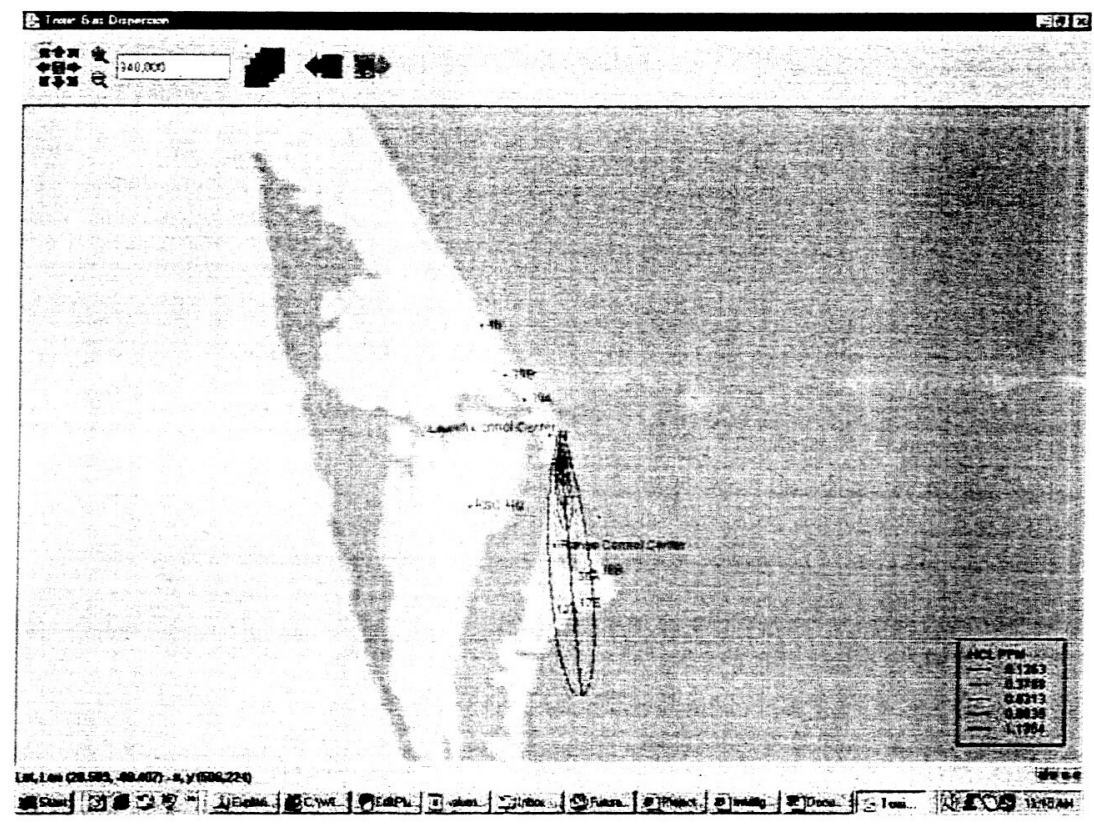

Figure 5. Contour plot of gas concentration

JMenuBar. This extension is the implementation of the MapHandlerChild methods that let it use the MapHandler to find MenuBarMenus which will be added to the MenuBar in the order in which they are found. The layerPanel is used to manage the display and order of the layers in the MapBean. Layers are components that get added to the MapBean in a hierarchical stacking order. The map is drawn by painting the graphics of each 
layer starting with the bottom most one and proceeding up the hierarchy. Successive layers render their graphics on top of the graphics of lower ones. In figure (6), multiple layers are overlayed. PlugIns are components that are used by the PlugInLayer to fetch data and prepare graphics for the map. The OMGraphics package provides a simple way to construct vector and raster graphics out of geo-spatial and XY data. The Kennedy space center location details are used to plot in the map by OMGraphics. When a layer is added to the MapBean, it automatically becomes a ProjectionListener of the MapBean. This means that the layer receives notification when the projection of the map changes. The projection interface allows read-only access to the current MapBean projection. The MapBean updates all the Layers and other projectionListeners when the view changes.

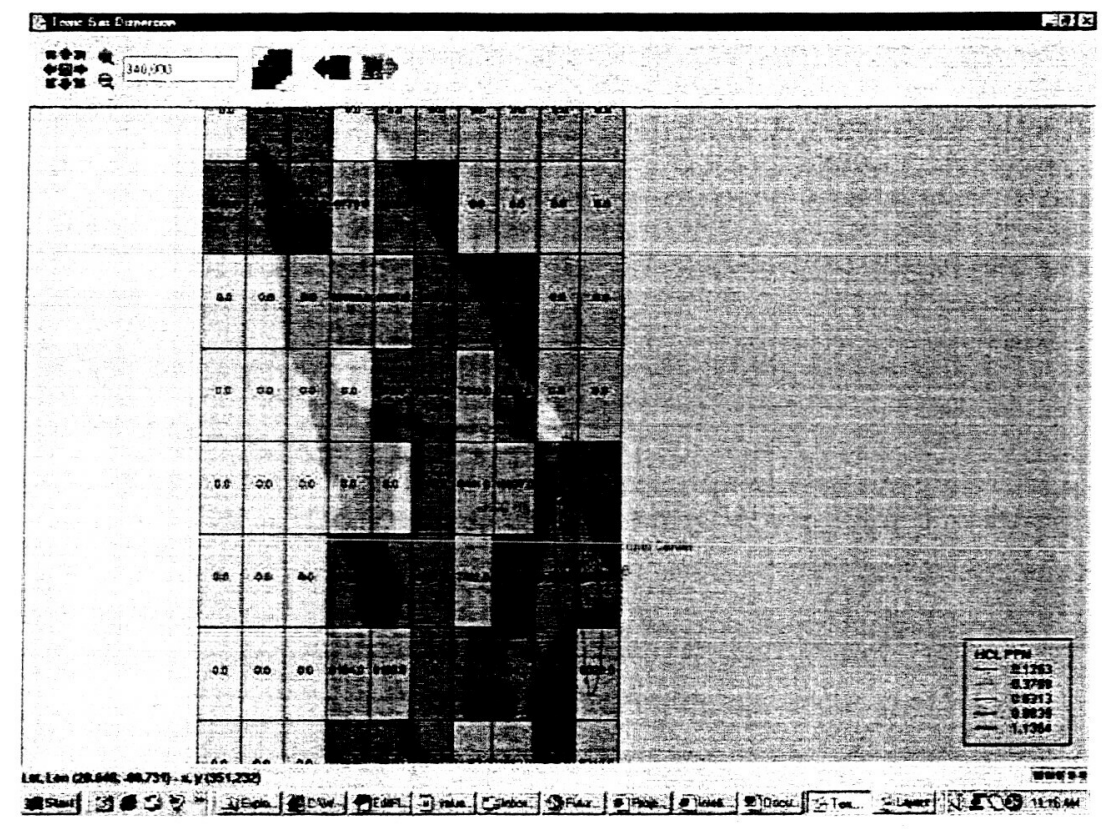

Figure 6. Layer of population grid over chemical contour.

The population grid is computed by $10 \times 10$ grid over region of interest. The population is summed within a grid and it represents a population density of the grid. Once the population grid and chemical contour are computed, human health risk can be estimated using the hazard quotient. In the hazard quotient method, an individual normal adult hazard quotient index is calculated. When we express the risk to the exposed population, it is computed using the equation $8 .^{11}$

$$
\text { Risk to exposed population }=\text { Individual risk } \times \text { number in exposed population }
$$

The population grid and hazard quotients are used to compute the human health risk assessment of normal adults and risk contour are drawn. Based on launch commit criteria, the expected casualty and risk criteria are tabulated in following table 3. The figure 7 shows risk contour in four levels. The risk contours represent four levels of risks for a particular chemical during launch. The inner contour represents the maximum vulnerability to the chemical exhausts from rockets. If the values exceeds table 3 values, a 'No-go' situation arises. Go/No-go situation can be assessed for a particular toxic chemical concentration at the ground level and human health risk based on table 3 .

\section{CONCLUSION}

The human health risk assessment model, toxic gas dispersion model coupled with GIS capability over the web and dissemination of information to all centers in real time is the great advantage of this web based model. 
Table 3. Risk Criteria.

\begin{tabular}{|l|c|c|}
\hline Population Group & $\begin{array}{c}\text { Expected Casualty } \\
\text { Cumulative }\end{array}$ & Individual \\
\hline General Public & $30 \times 10^{-} 6$ & $1 \times 10^{-} 6$ \\
\hline Mission Essential & $300 \times 10^{-} 6$ & $10 \times 10^{-} 6$ \\
\hline
\end{tabular}

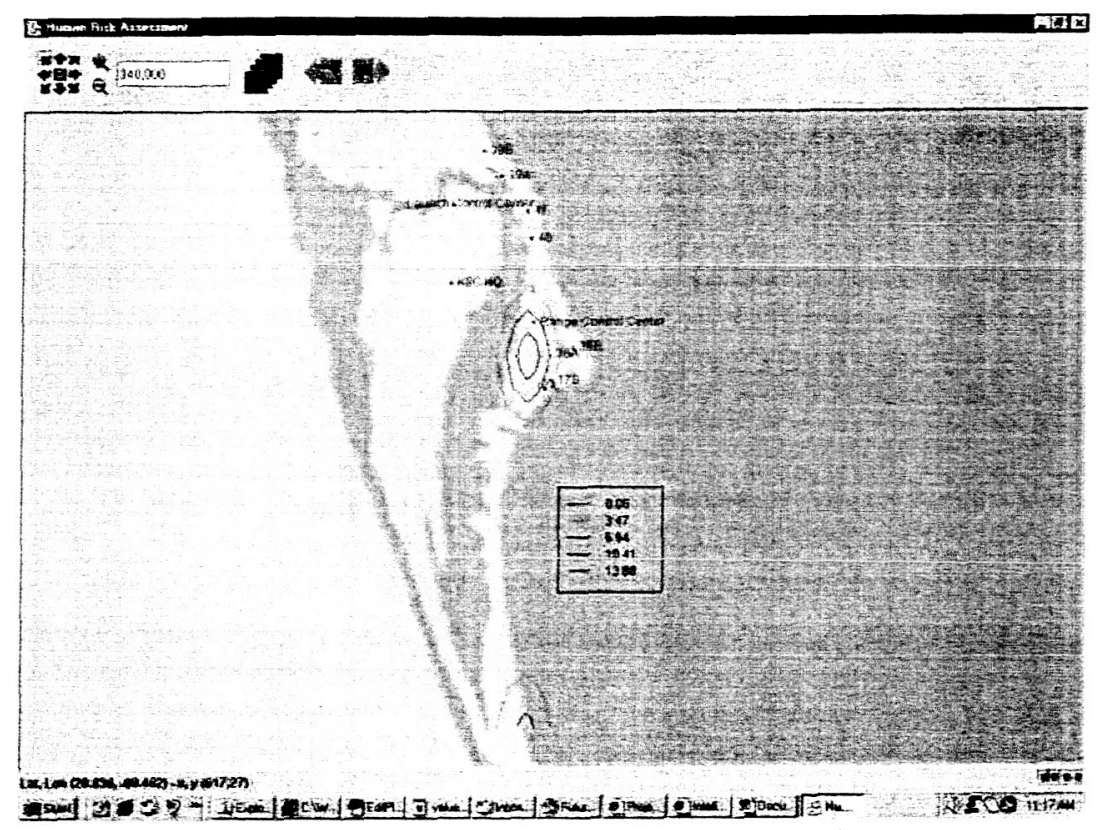

Figure 7. Risk contour for normal adults.

Different scenarios for various toxic chemicals and risk assessment can be generated remotely. Presently the model can be applied to normal adult population near launch areas. Future enhancement will include sensitive populations, multiple chemical exposure and path ways will be incorporated in the model.

\section{ACKNOWLEDGMENTS}

We would like to acknowledge useful comments, suggestions and discussions from Deepak Kulkarni and Francis Enomoto. The authors wish to thank Dr. Michael Shafto and Dr. Butler Hine for their financial support through CICT program at NASA Ames Research center.

\section{REFERENCES}

1. USEPA, The risk assessment guidelines of 1986, Office of Health and Environmental assessment USEPA, Washington DC 20460, 1986.

2. NRC, Assessment of exposure-response functions for rocket-emission toxicants, National Academy Press, 1998.

3. J. Bjorklund, R. Dumbauld, C. Cheney, and H. Geary, "Gas dispersion model," in User's manual for the REEDM (Rocket Exhaust Effluent Diffusion Model), H. C. C. Inc., ed., pp. 1-40, NASA, 1982. 
4. J. Hudson, A. See, and L. Philipson, "Launch area toxic risk analysis (latra): Risk management program," Proc. JANNAF 28th Propellant development and Characterization subcommitte and 17th Safety and Environmental protection subcommitte joint meeting, pp. 183-191, 1999.

5. R. Bennett and A. McDonald, "Local environmental and toxicity issues for rcoket launching and testing," Proc. JANNAF 28th Propellant development and Characterization subcommitte and 17th Safety and Environmental protection subcommitte joint meeting, pp. 153-173, 1999.

6. L. Philipson, J. Hudson, and A. See, "Exposure-response functions in air force toxic risk modeling," Toxicology 111, pp. 239-249, 1996.

7. BBN-Technologies, "http://openmap.bbn.com," 1992.

8. G. Briggs, "Some recent analysis of plume rise observations," Proc. Second International Clean Air Congress, pp. 6-11, 1970.

9. M. R. Beychok, Fundamentals of Stack Gas Dispersion, Milton Beychok, Irvine, California, 1997.

10. Florida-State-University, "http://www.met.fsu.edu/cudos/rawins/," 1998.

11. A. Yassi, T. Kjellstrom, T. deKok, and T. Guidotti, Basic Environmental Health, World Health Organization, 1998. 